\title{
DENOMINACIONES PARA TRASTORNOS MENTALES CONOCIDAS POR ESTUDIANTES DE MEDICINA: UN ESTUDIO CUALITATIVO
}

\author{
Adalberto Campo-Arias $^{1 *}$, Guillermo A. Ceballos-Ospino ${ }^{2}$ y Edwin Herazo $^{3}$ \\ ${ }^{1,2}$ Universidad del Magdalena, Santa Marta, Colombia \\ ${ }^{3}$ Instituto de Investigación del Comportamiento Humano, Bogotá, Colombia \\ *Autor de correspondencia: acampoa@unimagdalena.edu.ve \\ Recibido Septiembre 2020; Aceptado Noviembre 2020
}

\begin{abstract}
Resumen - El objetivo del presente trabajo fue explorar las denominaciones conocidas para trastornos mentales en un grupo de estudiantes de medicina de Santa Marta, Colombia. Se diseñó un estudio cualitativo con estudiantes que iniciaban un curso de psiquiatría listaron palabras o frases escuchadas en la comunidad para denominar a las personas que reúnen criterios para un trastorno mental. Se realizó un análisis basado en teoría fundamentada. Participaron 21 estudiantes, 15 mujeres y 6 hombres entre 18 y 25 años. Se contaron 228 palabras que se reagruparon en 98 connotaciones similares: 40 correspondían términos peyorativos populares: loco o chiflado. Basados en los resultados se concluye que es alto el número de denominaciones para los trastornos mentales conocidas por este grupo estudiantes de medicina. Estas palabras generalmente tienen una connotación peyorativa.
\end{abstract}

Palabras clave: Prejuicio, trastornos mentales, estudiantes de medicina, teoría fundamentada, estudios cualitativos.

\section{DENOMINATIONS FOR MENTAL DISORDER RECOGNIZED BY MEDICAL STUDENTS: A CUALITATIVE RESEARCH}

\begin{abstract}
The present work's objective was to explore the recognized denominations for mental disorders in a group of medical students. A qualitative study was done. Students who started a psychiatry course were asked the words or phrases heard in the community to name people who meet mental disorders criteria. The analysis was based on grounded theory. A total of 21 students participated, 15 women and 6 men, between 18 and 25 years. A total of 228 words or phrases were counted that were grouped into 98 similar connotations. The most frequent denomination was crazy. Based on the results, it is concluded that the number of recognized names for mental disorders in this group of medical students is high. These words generally have a pejorative connotation.
\end{abstract}

Keywords: Prejudice, mental disorders, medicine students, grounded theory, qualitative studies.

\section{Introducción}

El lenguaje cotidiano, jerga o argot juega un papel en la comunicación diaria (Granello y Gibbs, 2016) y el lenguaje participa en el proceso de generación del estigma (Burgers y Beukeboom, 2016). Algunas nominaciones comunes pueden tener connotaciones negativas y usadas peyorativamente para discriminar a 
otras personas (Alarcón, 2017). El empleo de denominaciones peyorativas es frecuente para nombrar las personas que reúnen criterios para trastornos mentales (Rose et al., 2007).

Las denominaciones para los trastornos mentales cobran importancia según el contexto, los prejuicios y las experiencias con personas que reúnen criterios para trastornos mentales (Sheenan et al., 2017). En el proceso de consolidación de las denominaciones peyorativas en las comunidades los medios de comunicación juegan un papel determinante al exagerar algunos rasgos desfavorables que ocasionalmente se pueden presentar en un trastorno mental (Smith, 2018).

Se entiende por complejo estigma-discriminación (CED) la relación entre estigma, prejuicio, estereotipo y discriminación (Pescosolido y Martin, 2015; Tyler y Slater, 2018). El estigma es la connotación negativa que se da a cualquier característica; prejuicio es una idea negativa que se resiste la modificación pese a las observaciones contrarias; estereotipo es el prejuicio socialmente compartido; y la discriminación son las acciones que vulneran derechos basada en un estigma (Campo-Arias y Herazo, 2015; Tyler y Slater, 2018). Los trastornos mentales pueden afectar a cualquier individuo en algún momento de la vida (BarreraHerrera et al., 2019). Los estudiantes de medicina con frecuencia pueden reunir criterio para cualquier trastorno mental (Cuttilan et al., 2016; Rotenstein et al., 2016). Sin embargo, la solicitud de ayuda profesional en salud mental puede estar condicionada por el CED relacionado con trastornos mentales (CEDRTM) (Gyamfi et al., 2018).

No obstante, el número creciente de acciones orientadas a la reducción del CEDRTM (Mascayano et al., 2015; Thornicroft et al., 2016),el CEDRTM está presente en la población general (Karaca et al., 2019).El CEDRTM se evidencia en el número de denominaciones peyorativas para las personas que reúnen criterios para un trastorno mental. Por ejemplo, en estudiantes de secundaria, Rose et al observaron el uso de 250 denominaciones para describir a una persona con trastorno mental; esas denominaciones las agruparon en términos despectivos populares, estados emocionales negativos, confusión entre discapacidades físicas, dificultades de aprendizaje y problemas de salud mental, diagnósticos psiquiátricos y términos asociados a violencia (Alarcón, 2017).

Asimismo, es frecuente observar alta frecuencia de CEDRTM en el sector sanitario en estudiantes y profesionales de la salud (James et al., 2012). En el ámbito universitario, la actitud de los estudiantes de medicina no siempre es la más favorable hacia los trastornos mentales (Shehata y Abdeldaim, 2020). No obstante, se desconocen estudios que presenten los términos para trastornos mentales comúnmente usados en español por niños, adolescentes o adultos. Conocer estos términos es importante porque reflejan un patrón cultural general que implica CEDRTM (Burgers y Beukeboom, 2016; Granello y Gibbs, 2016).

El CEDRTM tiene impacto negativo en los pacientes y las familias. El malestar general asociado al CEDRTM es mayor que el debido al trastorno mental mismo (Campo-Arias y Herazo, 2015; Pescosolido y Martin, 2015; Tyler y Slater, 2018). El CEDRTM es un problema importante en la vida de los pacientes y se configura como un estresor más y con frecuencia representa la principal barrera de acceso a los servicios de salud mental (Campo-Arias et al., 2020).

El objetivo del presente estudio fue conocer las denominaciones populares para trastornos mentales conocidas en estudiantes de medicina de Santa Marta, Colombia. 
Denominaciones para trastornos mentales conocidas por estudiantes de medicina: un estudio cualitativo

\section{Materiales y métodos}

Se diseñó una investigación cualitativa. Se prefirió aproximación por teoría fundamentada porque explora al ser social en interacción con el medio cultural. La teoría fundamentada tiene raíces epistemológicas en la sociología y del interaccionismo simbólico y plantea que es posible obtener información sobre las opiniones e influencias sociales en la construcción de actitudes; pero, que los investigadores contribuyen a la formulación y construcción del conocimiento (De La Espriella y Gómez, 2020).

El interaccionismo simbólico es una forma de interpretación las realidades basado en distintas perspectivas que adquieren las personas en la construcción de una realidad particular. El objetivo de la teoría fundamentada es la formulación de teorías basadas en la observación y el análisis del quehacer humano individual y al interior de la comunidad (De La Espriella y Gómez, 2020).

\section{Población}

Se tomó una muestra intencional (Boddy, 2016). En el aula de clases, se solicitó la participación voluntaria a estudiantes de medicina que realizaban un taller para abordar el CEDRTM. El grupo iniciaba un curso de psiquiatría clínica en una universidad de Santa Marta, Colombia. En la Tabla 1 se presenta el número de inscritos en el curso, se observa que se contó con el 87,5\% de participación lo cual es aceptable para este tipo de estudios, con una muestra mayor de 16 personas.

Tabla 1. Población sometida a estudio

\begin{tabular}{|c|c|}
\hline $\begin{array}{c}\text { Número de Estudiantes curso } \\
\text { de psiquiatría }\end{array}$ & Cantidad \\
\hline Inscritos & 24 \\
\hline No participaron & 3 \\
\hline Total de la población & 21 \\
\hline
\end{tabular}

Fuente: Elaboración propia (2020)

De los 24 estudiantes inscritos en el curso, participaron 21 estudiantes, 15 mujeres $(71,4 \%$ ) y 6 hombres (28,6\%). Las edades de los estudiantes se observaron entre 18 y 25 años $(M=21,6 ; D E=1,6)$. Con relación a las características del lugar de la crianza, 15 estudiantes informaron su crianza en zona urbana (71,4\%) y 6 estudiantes en zonas de características rurales (28,6\%). En lo concerniente a la experiencia clínica en el área de psiquiatría, 18 estudiantes (85,7\%) iniciaban el curso por primera vez y 3 estudiantes $(14,3 \%)$ lo habían iniciado con alguna anterioridad. Un estudiante no asistió a la clase el día del taller y dos estudiantes no firmaron el consentimiento informado para incluir en el análisis.

\section{Técnica e instrumento para la recolección de datos}

En forma manual, se listaron todas las denominaciones. No se usaron preguntas generadoras. Se realizó una introducción general sobre el objetivo general del estudio, se dieron instrucciones para responder con lápiz y papel, y anotaran el mayor número de palabras conocidas, todas las palabras eran válidas, sin que ello indujera o condicionara las respuestas de los participantes. 


\section{Análisis de los datos}

Luego de listar manualmente las denominaciones dadas, se pasó a la codificación que incluyó la conceptualización; posteriormente, se llevó a cabo la reducción y el establecimiento de categorías para las denominaciones en término de propiedades o dimensiones y, finalmente, se agruparon las categorías en dominios emergentes (De La Espriella y Gómez, 2020).

Los tres autores en forma independiente realizaron la 'codificación abierta' para una exploración inicial de las denominaciones y la identificación de 'códigos preliminares'. Posteriormente, se realizó una 'codificación axial' en la que se reorganizaron los códigos identificados y, al final, se identificaron ‘códigos conceptuales'. De ese grupo de 'códigos conceptuales' llegó a las 'categorías' finales (De La Espriella y Gómez, 2020).

\section{Resultados y discusión}

Se recogieron de 228 palabras o términos que se reagruparon en 98 denominaciones o connotaciones similares. La denominación más frecuente fue loco, todos los estudiantes la incluyeron y, adicionalmente, un estudiante usó la palabra equivalente en inglés (crazy). Seguida por "le falta un tornillo o pieza” y "se le corrió la teja”, en sendas ocho ocasiones.

De las 98 denominaciones, 40 correspondían términos populares peyorativos (loco o chiflado); 26 términos correspondían a palabras de uso médico, como síntomas o diagnósticos formales (depresivo, o psicótico); 6 términos connotaban en particular al consumo de sustancias (alcohólico, o drogadicto); 6 términos aludían a comportamientos con implicaciones legales (bandido o pervertido); 5 denominación relacionadas con discapacidad intelectual (retrasado o mongólico); 4 denominaciones con la productividad monetaria (desechable o inservible); 4 al posible origen de los trastornos mentales (embrujado o endemoniado) y 7 incluye una variedad de términos, todos con implicaciones sociales negativas (agresivo o guache).La tabla 2 muestra ocho categorías que muestra las denominaciones más mencionadas, en orden alfabético, por los estudiantes. Los términos populares (loco) fueron más relatadas, hasta términos que podrían parecer exóticos como "guache" y podría reflejar más características de personalidad. Es llamativa la atribución a fuerzas paranormales, incluidas religiosas, para las causas o posibles explicaciones para los trastornos mentales.

Tabla 2. Principales categorías para las denominaciones.

\begin{tabular}{|c|c|c|c|c|c|c|c|}
\hline Populares & Técnicas & $\begin{array}{c}\text { Consumo de } \\
\text { sustancias }\end{array}$ & Delincuencia & $\begin{array}{c}\text { Discapacidad } \\
\text { intelectual }\end{array}$ & $\begin{array}{c}\text { Productividad } \\
\text { monetaria }\end{array}$ & $\begin{array}{c}\text { Causas } \\
\text { paranormales }\end{array}$ & Otros \\
\hline Crazy & Bipolar & Alcohólico & Antisocial & Discapacitado & Desechable & Castigo divino & Agresivo \\
\hline Chiflado & Depresivo & Drogadicto & Bandido & Especial & Inservible & Embrujado & Guache \\
\hline Loco & Esquizofrénico & Embalado & Pervertido & Mongólico & Mantenido & Endemoniado & Manipulador \\
\hline Sollado & Psicótico & Fumado & Psicópata & Retrasado & Vagabundo & Poseído & Posudo \\
\hline
\end{tabular}

Fuente: Elaboración propia (2020)

En el presente análisis se observaron siete categorías principales para las denominaciones comunes que se dan a las personas que reúnen criterios para un trastorno mental: términos peyorativos que aluden al consumo de sustancias legales o ilegales, comportamientos ilegales, discapacidad intelectual, productividad monetaria y relacionada con posibles etiologías de los trastornos mentales. Todas con 
connotaciones negativas. Los estudios para comparar estos hallazgos son escasos. Sin embargo, se pueden coincidir, dada las particularidades de los idiomas, con los resultados de Rose et al en estudiantes ingleses de 14 años; estos mencionaron 250 denominaciones para trastorno mental que se agruparon en cinco categorías: términos peyorativos populares, relacionados con estados emocionales negativos, una mezcla entre discapacidades físicas, dificultades de aprendizaje y problemas de salud mental, el uso de términos propios del contexto clínico psiquiátrico y, por último, terminología relacionada con comportamientos violentos (Rose et al., 2007).

El número de denominaciones sugiere que el CEDRTM es muy frecuente en la población general colombiana, como en otros países africanos, asiáticos y europeos (Karaca et al., 2019). No obstante, la existencia de normas legales en Colombia específicas que prohíben todo tipo de discriminación, en la práctica algunas observaciones heurísticas dan a entender que el CEDRTM no se limita sólo a los pacientes, sino también se extiende a familiares y profesionales de la salud mental (Yanos et al., 2020).

Asimismo, sale a relucir que una simple la legislación no es suficiente para cambiar los imaginarios colectivos. Y que los tecnicismos se pueden usar en el contexto no médico con connotaciones negativas. Esta observación puede sugerir que el incremento en el conocimiento en trastornos mentales por la comunidad no necesariamente cambia las actitudes y las acciones discriminatorias se mantienen en el lenguaje cotidiano (Rose et al., 2007). En el contexto de la educación médica, los estudiantes de medicina con frecuencia informan bromas que hacen los profesores de otras especialidades médicas sobre los pacientes y los profesionales de la psiquiatría. Esto puede ser una explicación para la persistencia del CEDRTM en estudiantes de medicina (McManus y Sproston, 2000; Sternszus y Cruess, 2016).

El aspecto en que se relaciona el CEDRTM con la productividad monetaria ausente o disminuida, hay que entenderla en el contexto de un modelo de sociedad caracterizado por la acumulación de capital como un factor de reconocimiento de éxito, ligado igualmente a un desempeño laboral de ascenso permanente. No merece respeto la persona que no puede trabajar. La capacidad para trabajar y producir un enfoque más amplio están incorporadas en la definición de salud mental de la Organización Mundial de la Salud y la contemplada en la Ley 1616 de 2013 de Colombia (Ley 16, 2013; OMS, 2004).

La reducción del CEDRTM necesita intervenciones en múltiples niveles y sectores, más allá que la simple educación o incremento del conocimiento sobre los trastornos mentales no sólo en la comunidad general, sino también en estudiantes de medicina, para favorecer la aceptación y no exclusión de las personas que reúnen criterios para un trastorno mental (Burgers y Beukeboom, 2016; Gyamfi et al., 2018). El CEDRTM es un estresor adicional y representa una de las principales barreras de acceso a los servicios de salud mental; es evidente que las personas evitan hacer parte de un grupo valorado negativamente por la sociedad, es decir, que el CEDRTM representa un porcentaje importante de la carga de enfermedad explicada por los trastornos mentales y no sólo afecta la salud mental, sino también la salud física de las personas.(Campo-Arias et al., 2020). Los medios de comunicación constituyen una fuente importante de información que influye en la audiencia, por lo tanto, la representación que hacen de las personas que reúnen criterios para trastornos mentales refuerza los imaginarios y estereotipos alrededor de los trastornos mentales (Smith, 2018).

Este estudio es un aporte al conocimiento del CEDTRM para países de habla hispana dado que las denominaciones populares suelen variar según el idioma, y dentro de cada idioma según las regiones. No 
obstante, tiene la limitación propia de un estudio cualitativo que no permite generalizaciones más allá de los participantes y evitó indagar directamente las denominaciones usadas por los estudiantes fuera del contexto académico porque se quiso evitar la deseabilidad social.

\section{Conclusiones}

Se concluye que es alto el número de denominaciones para los trastornos mentales conocidas por este grupo estudiantes de medicina. Estas palabras generalmente tienen una connotación peyorativa y, posiblemente, afecta la búsqueda que ayuda de las personas cuando presentan síntomas psicológicos que ameritan evaluación y seguimiento en salud mental. Se necesitan intervenciones integrales para reducir el CEDRTM, con la inclusión de estudiantes de medicina, importante aumentar el conocimiento sobre trastornos mentales poblaciones, probablemente, la educación en salud mental, reduzca CEDRTM y con ello reducir el impacto negativo asociado a trastornos mentales en pacientes, familias y la sociedad.

\section{Referencias}

Alarcón, R. D. (2017). Estigma en la práctica psiquiátrica de un hospital general. Revista Médica Clínica Las Condes, 28(6), 818-825. https://doi.org/10.1016/j.rmclc.2017.09.001

Barrera-Herrera, A., Neira-Cofré, M., Raipán-Gómez, P., Riquelme-Lobos, P., \& Escobar, B. (2019). Apoyo social percibido y factores sociodemográficos en relación con los síntomas de ansiedad, depresión y estrés en universitarios chilenos. Revista de Psicopatología y Psicología Clínica, 24(2), 105-115. https://doi.org/10.5944/rppc.23676

Boddy, C. R. (2016). Sample size for qualitative research. Qualitative Market Research, 19(4), 426-432. https://doi.org/10.1108/QMR-06-2016-0053

Burgers, C., \& Beukeboom, C. J. (2016). Stereotype transmission and maintenance through interpersonal communication: The irony bias. Communication Research, 43(3), 414-441. https://doi.org/10.1177/0093650214534975

Campo-Arias, A., Ceballos-Ospino, G.A. \& Herazo, E. (2020). Barriers to access to mental health services among Colombia outpatients. International Journal of Social Psychiatry,66(6),600-606. https://doi.org/10.1177/0020764020925105

Campo-Arias, A. \& Herazo, E. (2015). El complejo estigma-discriminación asociado a trastorno mental como factor de riesgo de suicidio. Revista Colombiana de Psiquiatría, 44,243-250. https://doi.org/10.1016/j.rcp.2015.04.003

Cuttilan, A.N., Sayampanathan, A.A. \& Ho, R.C.M. (2016). Mental health issues amongst medical students in Asia: a systematic review [2000-2015]. Annals of Translational Medicine, 4,72. https://doi.org/10.3978/j.issn.2305$\underline{5839.2016 .02 .07}$

De La Espriella, R. \& Gómez, C. (2020). Teoría fundamentada. Revista Colombiana de Psiquiatría, 49(2), 127-133. https://doi.org/10.1016/j.rcp.2018.08.002

Granello, D. H., \& Gibbs, T. A. (2016). The power of language and labels:“the mentally ill” versus "people with mental illnesses”. Journal of Counseling \& Development, 94(1), 31-40. https://doi.org/10.1002/jcad.12059

Gyamfi, S., Hegadoren, K. \& Park, T. (2018). Individual factors that influence experiences and perceptions of stigma and discrimination towards people with mental illness in Ghana. International Journal of Mental Health Nursing, 27(1), 368377. https://doi.org/10.1111/inm.12331 


\section{Denominaciones para trastornos mentales conocidas por estudiantes de medicina: un estudio}

James, B.O., Omoaregba, J.O. \& Okogbenin, E.O. S. (2012). Stigmatising attitudes towards persons with mental illness: a survey of medical students and interns from Southern Nigeria. Mental Illness,4, e8. https://doi.org/10.4081/mi.2012.e8

Karaca, A., Acikgoz, F.,\&Cangur, S. (2019). Attitudes of community-leading occupational groups towards mental illnesses: the sample of a city in western Turkey. Community Mental Health Journal,55,1377-1388. https://doi.org/10.1007/s10597-019$\underline{00441-5}$

Ley 1616 (2013). Por medio de la cual se expide la Ley de Salud Mental y se dictan otras disposiciones. Bogotá: Congreso de la República de Colombia.

Mascayano, F., Lips, W., Mena, C. \& Manchego, C. (2015). Estigma hacia los trastornos mentales: características e intervenciones. Salud Mental, 38,53-58. http://www.scielo.org.mx/scielo.php?script=sci_arttext\&pid=S0185$\underline{33252015000100008}$

McManus, I. C. \& Sproston, K. A. (2000). Women in hospital medicine in the United Kingdom: glass ceiling, preference, prejudice or cohort effect? Journal of Epidemiology and Community Health, 54(1), 10-16. http://dx.doi.org/10.1136/jech.54.1.10

Pescosolido, B.A. \& Martin, J.K. (2015). The stigma complex. Annual Review of Sociology,41,87-116. https://doi.org/10.1146/annurev-soc-071312-145702

Rose, D., Thornicroft, G., Pinfold, V. \& Kassam, A. (2007). 250 labels used to stigmatise people with mental illness. BMC Health Services Research,7,97. https://doi.org/10.1186/1472-6963-7-97

Rotenstein, L.S., Ramos, M.A., Torre, M., Segal, J.B., Peluso, M.J., Guille, C., Sen, S. \& Mata, A. (2016). Prevalence of depression, depressive symptoms, and suicidal ideation among medical students: a systematic review and meta-analysis. Journal of the American Medical Association, 316(21),2214-2236. https://doi.org/10.1001/jama.2016.17324

Shehata, W.M. \& Abdeldaim, D.E. (2020). Stigma towards mental illness among Tanta University students, Egypt. Community Mental Health Journal, 56(3),464-470. https//doi.org/10.1007/s10597-019-00502-9

Sheehan, L., Nieweglowski, K. \& Corrigan, P. W. (2017). Structures and types of stigma. In The stigma of mental illness-end of the story? (pp. 43-66). Springer, Cham.

Smith, R.A. \& Applegate, A. (2018). Mental health stigma and communication and their intersections with education. Communication Education, 67(3),382-393. https://doi.org/10.1080/03634523.2018.1465988

Sternszus, R. \& Cruess, S.R. (2016). Learning from role modelling: making the implicit explicit. Lancet, 387,1257-1258. https:/doi.org/10.1016/S0140-6736(16)00381-0

Thornicroft, G., Deb, T. \& Henderson, C. (2016). Community mental health care worldwide: current status and further developments. World Psychiatry, 15(3), 276-286. https://doi.org/10.1002/wps.20349

Tyler, I. E., \& Slater, T. (2018). Rethinking the sociology of stigma. The Sociological Review, 66(4),721-743. https://doi.org/10.1177/0038026118777425

Yanos, P., DeLuca, J., Salyers, M., Fischer, M., Song, J., \& Caro, J. (2020). Cross-sectional and prospective correlates of associative stigma among mental health service providers. Psychiatric Rehabilitation Journal, 43(2), 85-90. https://doi.org/10.1037/prj0000378 